\title{
A New Idea for an Experimental Search for $\mu-e$ Conversion
}

\author{
Masaharu Aoki* on behalf of DeeMe Collaboration \\ Osaka U. \\ E-mail: aokimephys.sci.osaka-u.ac.jp
}

\begin{abstract}
A new experiment searching for $\mu$-e conversion by fully utilizing a high-power pulsed proton beam available at J-PARC Material and Life Science Facility (MLF) was proposed. The muonic carbon atom formation rate in a muon target of MLF is estimated to be $10^{10} / \mathrm{sec}$ for $1 \mathrm{MW}$ operation of a J-PARC booster. With this high formation rate of the muonic atoms, it is possible to perform a competitive search for $\mu$-e conversion search at the level of $10^{-14}$, nearly two orders of magnitude below current limits. A new secondary beam line will be dedicated to extract 105$\mathrm{MeV} / \mathrm{c}$ electrons from the muon production target. This beam line is compatible with a new muon g-2 experiment also planned at MLF.
\end{abstract}

35th International Conference of High Energy Physics

July 22-28, 2010

Paris, France

${ }^{*}$ Speaker. 
Any processes with Charged Lepton Flavor Violation (CLFV) are forbidden in the Standard Model $a$ priori. The higher order effect coming from the neutrino oscillation is suppressed by a GIM-like mechanism to the level of $10^{-50}$,which is far beyond the experimental accessibility. Therefore, the experimental observation of CLFV process is a clear proof of the physics beyond the Standard Model.

The latest experiment to search for $\mu^{-}-e^{-}$conversion was performed by SINDRUM II collaboration and gave the upper limit, $<7 \times 10^{13}[1]$, for the gold target. There are many CLFV processes other than $\mu^{-}-e^{-}$conversion, but none of them found CLFV signals yet[2]. On the other hand, there are numerous theoretical models giving predictions for the branching ratios of CLFV processes [3]. A CLFV signal may be seen by experiments which improve the current limits, and it is conceivable that the CLFV signal lies waiting to be discovered right under the current limit. A new experiment searching for $\mu^{-}-e^{-}$conversion process at the $10^{-14}$ level would be a highly competitive addition to the field.

\section{A New Idea for the Experimental Search}

DeeMe is a newly proposed experiment to search for the $\mu$-e conversion at J-PARC MLF by using 3$\mathrm{GeV}$ pulsed proton beam. The experiment utilizes muonic atoms formed in a primary proton target, and extracts $105-\mathrm{MeV} / c$ electrons from the $\mu-e$ conversion by using a conventional secondary beamline. The number of muonic atoms formed in the primary target is estimated to be $10^{10} / \mathrm{sec}$ for $1 \mathrm{MW}$ operation of a J-PARC booster. Figure 1 shows the layout of the proposed secondary beamline. DeeMe aims to reach $10^{-14}$ of the single event sensitivity for silicon target with this beamline and with a silicon-carbide production target.

This beamline is designed so that it can be used for not only DeeMe but also other experiments such as muon $g-2$, muonium hyperfine structure, muoniumantimuonium conversion and so on. Fruitful outcomes of the muon fundamental science will be expected from J-PARC MLF in near future.

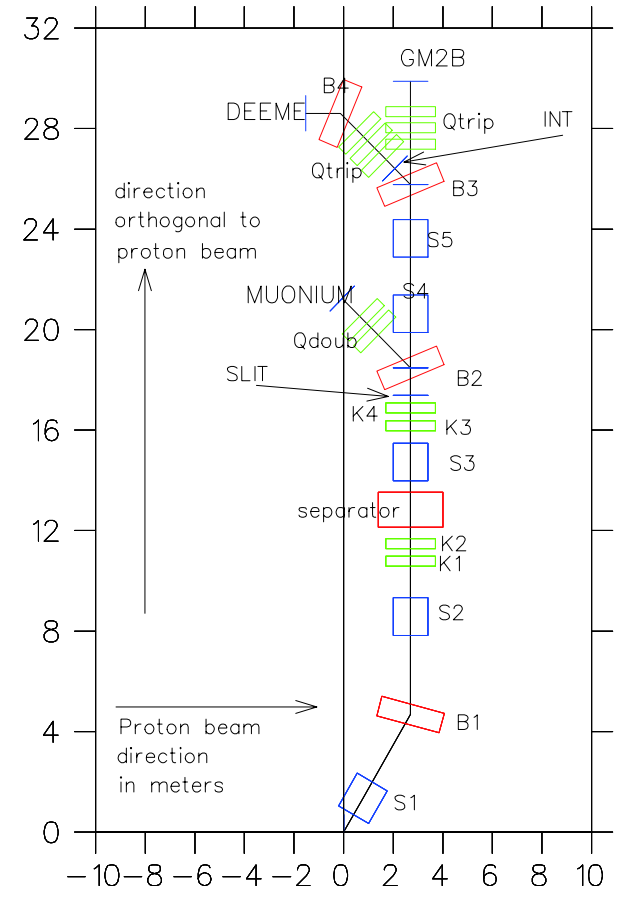

Figure 1: DeeMe beamline.

\section{References}

[1] W. Burtl et al. (The SINDRUM II Collaboration), A search for $\mu$-e conversion in muonic gold, Eur. Phys. J. C 47 (2006) 337.

[2] W.J. Marciano et al., Charged Lepton Flavor Violation Experiments, Annu. Rev. Nucl. Part. Sci. 58 (2008) 315 .

[3] Y. Kuno and Y. Okada, Muon decay and physics beyond the standard model, Rev. Mod. Phys. 73 (2001) 151. 